\title{
Angle-Resolved and Resonant Photoemission Study of the Valence Bands of $\alpha$-La(0001) on W(110)
}

\author{
Yoshiharu Enta1 ${ }^{*}$, Osamu Morimoto ${ }^{2}$, Hiroo Kato', Yasuo Sakisaka1 \\ ${ }^{1}$ Graduate School of Science and Technology, Hirosaki University, Hirosaki, Japan \\ ${ }^{2}$ Spring-8 Service Co., Ltd., Tatsuno, Japan \\ Email: *enta@hirosaki-u.ac.jp
}

Received 17 December 2015; accepted 30 January 2016; published 3 February 2016

Copyright (C) 2016 by authors and Scientific Research Publishing Inc.

This work is licensed under the Creative Commons Attribution International License (CC BY). http://creativecommons.org/licenses/by/4.0/

c) (7) Open Access

\begin{abstract}
We report a photoelectron spectroscopic study of the valence bands of double hexagonal-closepacked (dhcp) $\alpha$-La(0001) films epitaxially grown on W(110) at room temperature. The La $5 d$ photoemission cross section in the photon energy region from $20 \mathrm{eV}$ to $130 \mathrm{eV}$ was obtained and the valence-band structure of $\alpha$-La was determined. Except for $4 f$-related structures, the valenceband structures of $\operatorname{dhcp} \alpha$-La and $\operatorname{dhcp} \beta$-Ce were found to resemble each other. From the band structure, the crystal structure of the La film was confirmed. No evidence for the existence of a $5 d$-like surface state near the Fermi energy at the $\bar{\Gamma}$ point of the surface Brillouin zone was obtained and a $6 s$ band bottom was identified.
\end{abstract}

\section{Keywords}

Lanthanum Thin Film, Electronic Structure, Valence Band, Tungsten Surface, Photoemission, Angle-Resolved Spectroscopy

\section{Introduction}

The unusual physical properties of the various phases of metallic La have been the subject of many experimental and theoretical investigations [1]-[8]. La exists in three different phases at atmospheric pressure: double hexagonal-close-packed (dhcp) $\alpha$ phase below $609 \mathrm{~K}$, fcc $\beta$ phase between $609 \mathrm{~K}$ and $1138 \mathrm{~K}$, and bcc $\gamma$ phase between $1138 \mathrm{~K}$ and its melting point $(1191 \mathrm{~K})$. Although the stable form of La below $609 \mathrm{~K}$ is the dhcp $\alpha$ phase, the free energy difference between the $\alpha$ and $\beta$ phases is so small that the fcc phase can coexist below $609 \mathrm{~K}$, in

\footnotetext{
${ }^{*}$ Corresponding author.
}

How to cite this paper: Enta, Y., Morimoto, O., Kato, H. and Sakisaka, Y. (2016) Angle-Resolved and Resonant Photoemission Study of the Valence Bands of $\alpha$-La(0001) on W(110). World Journal of Condensed Matter Physics, 6, 17-26. 
a metastable form, with the $\alpha$ phase.

The ground-state electronic configuration is $[\mathrm{Xe}] 4 f^{0} 5 d^{1} 6 s^{2}$ for La atom and can be denoted as [Xe $] 4 f^{0}(5 d 6 s)^{3}$ for La metal considering possible hybridization. Compared with the isoelectronic elements $\mathrm{Y}$ and Sc, which are not superconducting under atmospheric pressure, La has a high superconducting transition temperature $T_{\mathrm{C}}$ of $4.88 \mathrm{~K}$ (dhcp $\alpha$ ) and $6.05 \mathrm{~K}$ (fcc $\beta$ ). It is worth noting that the physical properties of La are quite unusual in the $\beta$ phase which is metastable below $\sim 609 \mathrm{~K}$ at atmospheric pressure, rather than in the stable $\alpha$ phase. Under pressure, $T_{\mathrm{C}}$ for the $\beta$ phase rises sharply from $\sim 6 \mathrm{~K}$ at ambient pressure and saturates at a value of $\sim 13 \mathrm{~K}$ around $2 \times$ $10^{7} \mathrm{~Pa}$. This rapid rise of $T_{\mathrm{C}}$ with pressure for fcc $\beta$-La is the most dramatic among all the elements. In addition, the temperature dependence of the thermal-expansion coefficient is quite anomalous for fcc $\beta$-La: it becomes negative at $\sim 40 \mathrm{~K}$, and reaches its largest negative value at $\sim 18 \mathrm{~K}$.

These remarkable properties have led to speculations and suggestions about their electronic origin that a mechanism involving $4 f$ electrons is responsible and the electronic wave functions at the Fermi level contain a significant admixture of $4 f$ character. However, bremsstrahlung-isochromat [9] and inverse photoemission [10] experiments indicate that the $4 f$ state in La lies $\sim 5 \mathrm{eV}$ above the Fermi level $\left(E_{\mathrm{F}}\right)$ and is $\sim 1 \mathrm{eV}$ wide. Therefore, it is generally considered that the $4 f$ hybridization with the occupied states is negligibly small and does not affect the ground-state properties such as lattice dynamics or superconductivity of fcc $\beta$-La.

Understanding the properties of $\mathrm{La}$ in relation to the electronic structure is, of course, important. Furthermore, in addition to its intrinsic interest, La is of importance as a reference system for the chemically similar $4 f$ metals. As the first member of the rare-earth series of elements, the properties of La are frequently compared with those of other rare earths to help interpret properties be associated with the occupied $4 f$ levels. The valence-band photoemission of Ce exhibits two peaks attributed to $4 f$ emission. To understand better the electronic properties of $\mathrm{Ce}$, it is useful to study the neighboring element La with no $4 f$ electrons. Several photoemission experiments have been done to determine the valence-band electronic structure of $\mathrm{La}$ [11]-[14], suggesting the existence of a surface state near $E_{\mathrm{F}}$ at the $\bar{\Gamma}$ point of the surface Brillouin zone (SBZ). The interest in surface states arises from the strong influence of the surface electronic structure on physical and chemical properties of metal surfaces. However, the valence-band photoemission investigation for La is still not satisfactory and incomplete, since only part of the bands was studied. It cannot be denied that there has been relatively little theoretical effort given to determine the electronic structure of La, although there are a large number of papers [15]-[22]. This is no doubt related to the scarcity of experimental data available for comparison.

In this paper, we report the results of angle-resolved (ARPES) and resonant valence-band photoemission of epitaxial thin films of La grown on W(110) using synchrotron radiation. Photon-energy $(h v)$ dependence of the intensities of the photoemission peaks is measured to characterize the energy bands of La and the $5 d 6 s$ valenceband structures of $\mathrm{La}(0001)$ film are determined.

\section{Experimental}

The experiments were performed at the beamline BL-3B of the Photon Factory, Institute of Materials Structure Science, High Energy Accelerator Research Organization (KEK). ARPES spectra were measured at room temperature using a hemispherical electron analyzer with an acceptance of $\pm 1^{\circ}$. Total instrumental energy resolution was $50-100 \mathrm{meV}$, depending on the photon energy $(h v)$ in the range of $20-130 \mathrm{eV}$. Each series of spectra was normalized to the relative flux of incident photons. The light incidence angle from the surface normal was fixed to be $45^{\circ}$.

The clean W(110) surface, having sharp $(1 \times 1)$ low-energy electron diffraction (LEED) patterns with low backgrounds, was prepared by repeated heating it to $\sim 2300^{\circ} \mathrm{C}$ in ultrahigh vacuum. The amounts of impurities were below the detection limit of Auger electron spectroscopy (AES).

La (purity 99.99\%) was deposited in situ on the $\mathrm{W}(110)$ surface by electron-beam evaporation, with the substrate held at room temperature, after a long outgassing of the La source. The base pressure in the experimental chamber was $1.3 \times 10^{-8} \mathrm{~Pa}$, rising to $4.2 \times 10^{-8} \mathrm{~Pa}$ during deposition. No traces of surface impurities including oxygen and carbon contaminations were found by AES. The films (up to 15 monolayers) grown under these conditions showed sharp hexagonal LEED patterns corresponding to the formation of well-ordered dhcp $\alpha$ $\mathrm{La}(0001)$ or $\mathrm{fcc} \beta$ - $\mathrm{La}(111)$ surfaces. The LEED patterns could not be further improved by sample annealing at $370-570 \mathrm{~K}$. The interplanar spacing of dhcp $\mathrm{La}(0001)$ (3.04 $\AA$ ) is almost equal to that of fcc $\mathrm{La}(111)(3.06 \AA)$, and therefore the distinguishing between dhcp La and fcc La phases is difficult in the LEED experiment. As 
stated above, the possibility of coexisting of fcc $\beta$-La in a metastable form cannot be denied, but our ARPES band dispersion data are consistent with the dhcp structure rather than the fcc structure. Therefore, throughout this paper, only the stable dhcp $\alpha$-phase below $609 \mathrm{~K}$ is referred. The amounts of deposits were determined from the Auger-peak-intensity ratio $I_{\mathrm{La}(78 \mathrm{eV})} / I_{\mathrm{W}(169 \mathrm{eV})}$, as described previously [23]. The thicknesses of La films are quoted below in units of equivalent monolayers (ML), representing the number of close-packed La layers of the dhcp $\mathrm{La}(0001)$ orientation by assuming uniform thickness.

\section{Results and Discussion}

Figure 1 shows normal-emission ARPES spectra of $\alpha-\mathrm{La}(0001) / \mathrm{W}(110)$ in the valence-band region measured at $h v=34 \mathrm{eV}$, as a function of the coverage of La (from $0 \mathrm{ML}$ to $5 \mathrm{ML}$ ). Each spectrum was measured for a newly deposited La film. The signal from the underlying W(110) substrate will be attenuated due to inelastic scattering of some of the photoelectrons as they traverse through the layer of La metal, depending on the thickness of the layer. The 0 -ML spectrum is reduced by 0.06 times considering attenuation of electrons due to inelastic scattering in 1.8-ML La. In Figure 1, two La-induced emissions are seen: one labeled $A$ is near $E_{\mathrm{F}}$ and the other labeled $B$ at $\sim 1.3-\mathrm{eV}$ binding energy $\left(E_{\mathrm{B}}\right)$. These emissions grow as increasing La deposition. Besides $A$ and $B$, a weak structure is also seen at $E_{\mathrm{B}} \sim 2 \mathrm{eV}$. This $2 \mathrm{eV}$ structure was found to decrease to zero with La deposition, and therefore we consider it a trace of underlying $\mathrm{W}$ emission.

To obtain information about the character of peaks $A$ and $B$, we examine the $h v$ dependence of ARPES spectrum of La. Figure 2 shows a series of normal-emission spectra of $\sim 4-\mathrm{ML} \alpha-\mathrm{La}(0001) / \mathrm{W}(110)$ in the $h v$ range from $20 \mathrm{eV}$ to $130 \mathrm{eV}$. In addition to peaks $A$ and $B$, a weak emission labeled $C$ is indistinctly observed at $E_{\mathrm{B}} \sim$ $3.5 \mathrm{eV}$. It is noted that peaks $A$ and $B$ are positioned at almost constant binding energies of about $0.2 \mathrm{eV}$ and 1.3 $\mathrm{eV}$ for all $h v$, that is, both peaks hardly show dispersion along the axis of $k_{\perp}$ (wave vector perpendicular to the surface) for the 4-ML thickness film. This suggests that the $k_{\perp}$ is not a good quantum number for La films with thickness less than $4 \mathrm{ML}$. In the case of Ni films on W(110), it has been reported that the wave vector $k_{\perp}$ becomes a good quantum number and the $k_{\perp}$ dispersion of Ni starts to develop only when film thickness exceeds 5 ML [24]. Although the energy positions of peaks $A$ and $B$ are independent of $h v$, the intensities depend strongly on $h v$. In order to gain some insight into character of the peaks, we examine $h v$ dependence of the intensities of the peaks $A$ and $B$.

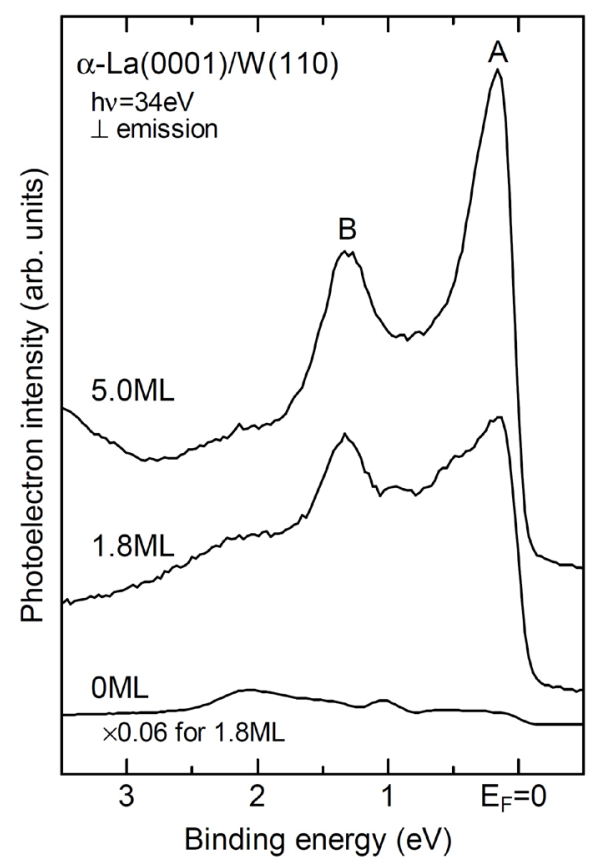

Figure 1. Normal-emission valence-band spectra of $\alpha-\mathrm{La}(0001) / \mathrm{W}(110)$, as a function of the coverage of La, measured at $h v=34 \mathrm{eV}$. The $0-\mathrm{ML}$ spectrum is a spectrum of W(110) substrate. 


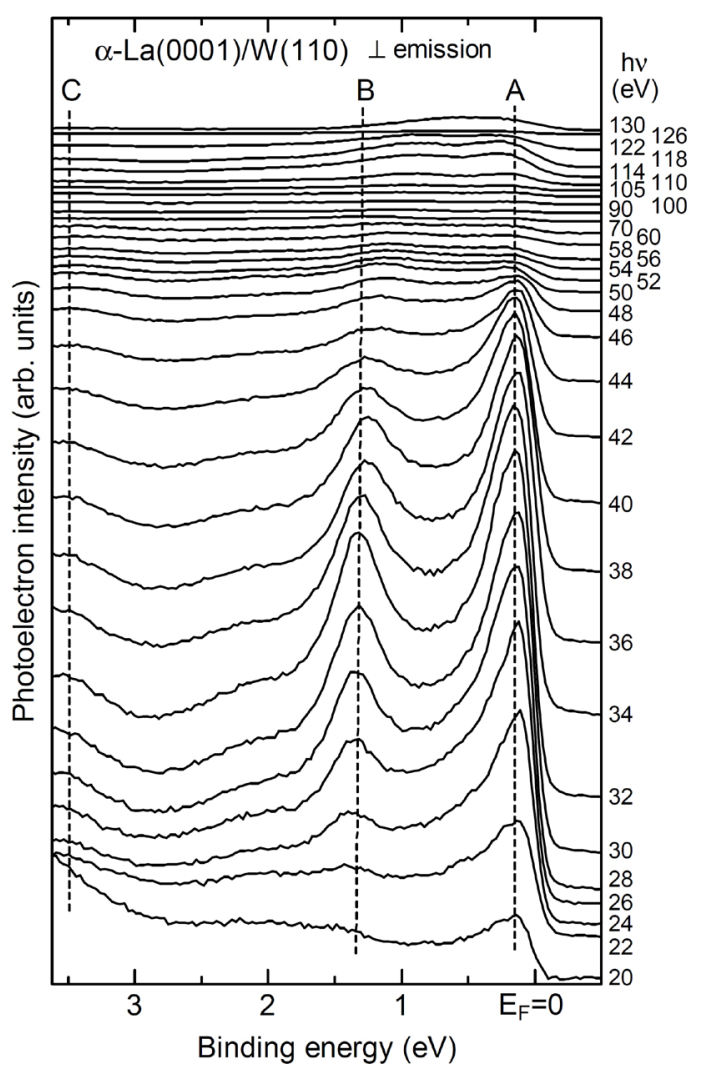

Figure 2. Normal-emission valence-band spectra of $\sim 4-\mathrm{ML} \alpha$ $\mathrm{La}(0001) / \mathrm{W}(110)$ as a function of $h v$ from $20 \mathrm{eV}$ to $130 \mathrm{eV}$.

Figure 3 shows $h v$ dependence of the heights (excitation spectra) of peaks $A$ and $B$ in the $h v$ region from 20 $\mathrm{eV}$ to $130 \mathrm{eV}$, obtained by averaging the data of Figure 2 and other two sets of similar series. The peak height was measured after properly subtracting a linear background from each spectrum. The heights of both peaks $A$ and $B$ rise up with increasing $h v$ to reach a maximum at $h v \sim 32 \mathrm{eV}$, thereafter falls monotonically to approach zero until $\sim 100 \mathrm{eV}$, and then, when $h v$ exceeds $\sim 100 \mathrm{eV}$, again increases to reach a second maximum centered at $h v \sim 116 \mathrm{eV}$. Now, we compare the excitation spectrum of La with that of Ce. The excitation spectrum of Ce has its own peculiar structure centered at $h v \sim 42 \mathrm{eV}$ [23]. This $42-\mathrm{eV}$ structure is due to the Ce $4 f$ electron photoemission. It should be noted that the present excitation spectrum of La does not have such a structure originated in $4 f$ electrons, supporting the previous conclusion that the $4 f$ hybridization with the occupied states is negligibly small in La. However, in the lower $h v$ region up to $\sim 100 \mathrm{eV}$, the excitation spectra for peaks $A$ and $B$ resemble that for Ce $5 d$ peak [23]. For $20 \mathrm{eV}<h v<40 \mathrm{eV}$, the Hartree-Fock-Slater atomic photoionization cross section for La $6 s$ is 10 - 30 times as small as that for La $5 d$ [25]. Thus, the profiles of the excitation spectra for peaks $A$ and $B$ indicate that their initial states are of La $5 d$ character. The $116-\mathrm{eV}$ structure is due to a two-step process: the La $4 d$ resonating giant photoexcitation $h v+4 d^{10}(5 d 6 s)^{3} \rightarrow 4 d^{9}(5 d 6 s)^{3} 4 f^{1}\left({ }^{1} \mathrm{P}_{1}\right)$ and the subsequent autoionization decay $4 d^{9}(5 d 6 s)^{3} 4 f^{1} \rightarrow 4 d^{10}(5 d 6 s)^{2}+e^{-}$(e denotes the ejected photoelectron), i.e., so-called $4 d$ resonant photoemission of La [26]-[29].

Figure 4 shows off-normal emission spectra of 4.0-6.0-ML $\alpha-\mathrm{La}(0001) / \mathrm{W}(110)$ as a function of the emission angle $\theta_{\mathrm{e}}$ along the $[10 \overline{1} 0](\bar{\Gamma}-\bar{M})$ azimuth. Three major peaks in the spectra are marked by tick marks and are labeled $A-C$ as above. From the spectra in Figure 4, we determined the $E$-versus- $k_{/ /}$dispersion $\left[E\left(k_{/}\right)\right]$along the $\bar{\Gamma}$ $\bar{M}$ direction of SBZ. The results are summarized in Figure 5. The four sets of band structures derived from Figures 4(a)-(d) are well in agreement.

As seen in Figure 5, the obtained band structure $E\left(k_{/ /}\right)$is rather complicated. Band $A$ is split into two bands at $k_{/ /} \sim 0.45 \AA^{-1}$ : the upper and lower bands are labeled $A 1$ and $A 2$, respectively. Peak $A 1$ is weak and shows substantially no $k_{/ /}$dispersion. As $k_{/ /}$increases up to $0.94 \AA^{-1}$, band $A 2$ disperses downward by $\sim 0.7 \mathrm{eV}$, to reach the 


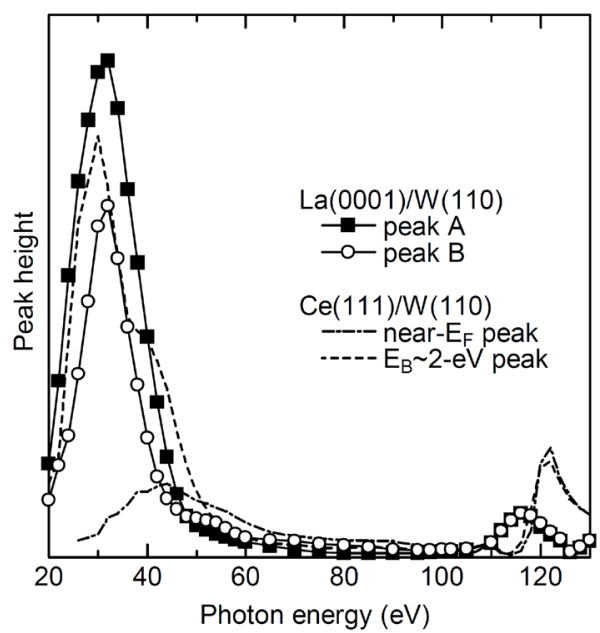

Figure 3. $h v$ dependence of the heights (excitation spectra) of the peaks $A$ and $B$, obtained by averaging the data of Figure 2 and other two sets of similar series. The excitation spectra of near- $E_{\mathrm{F}} 4 f^{1}$-final-state peak and $E_{\mathrm{B}} \sim 2$-eV $4 f^{0}$-final-state peak for $\gamma$-Ce(111) (from [23]) are shown to be compared with those of La. The Ce excitation spectra are normalized to the average of the $32-\mathrm{eV}$ maxima of peaks $A$ and $B$.
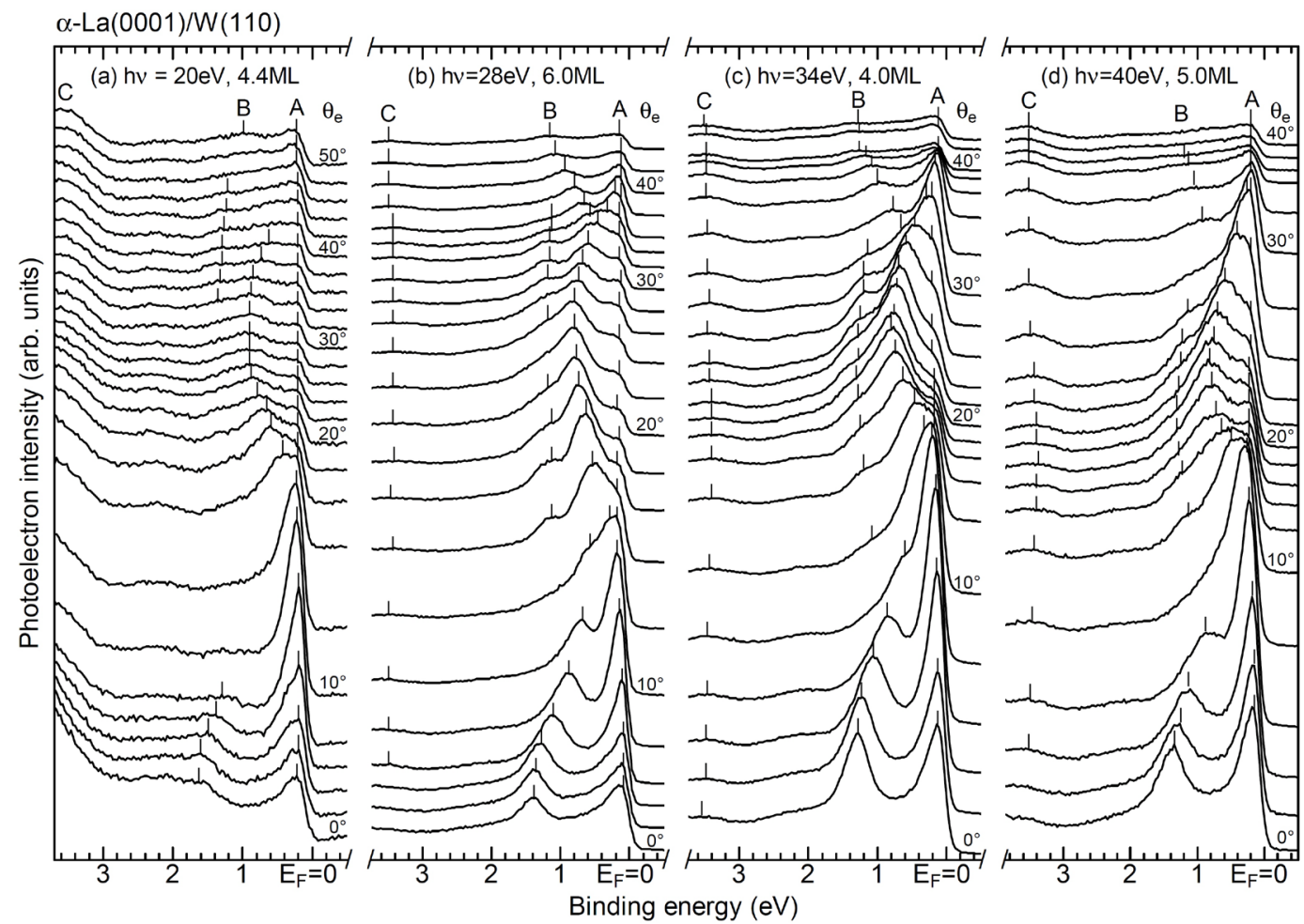

Figure 4. Off-normal emission valence-band spectra of $4.0-6.0-\mathrm{ML} \alpha-\mathrm{La}(0001) / \mathrm{W}(110)$ measured at (a) $h v=$ $20 \mathrm{eV}$; (b) $28 \mathrm{eV}$; (c) $34 \mathrm{eV}$; and (d) $40 \mathrm{eV}$, with the emission angle $\theta_{\mathrm{e}}$ changed by $2^{\circ}$ along the [10 $\left.\overline{1} 0\right](\bar{\Gamma}-$ $\bar{M}$ ) azimuth. The emission angle $\theta_{\mathrm{e}}$ is measured from the surface normal in the photoelectron collection plane.

bottoms at $E_{\mathrm{B}} \sim 0.85 \mathrm{eV}$. Thereafter, band $A 2$ disperses upward and bands $A 1$ and $A 2$ are merged into one at $k_{/ /} \sim$ $1.5 \AA^{-1}$. Band $B$ disperses upward as $k_{/ /}$increases up to $\sim 0.35 \AA^{-1}$ and then is split into two bands: upper band $B 1$ and lower band $B 2$. Thereafter, band $B 1$ still disperses upward until $\sim 0.5 \AA^{-1}$ and then downward: bands $B 1$ and $A 2$ unite to one labeled $A 2+B 1$ between $\sim 0.5$ and $\sim 1.35 \AA^{-1}$. Band $B 2$ disperses downward to reach the rather flat bottom at $E_{\mathrm{B}} \sim 1.27 \mathrm{eV}$ between $\sim 0.6$ and $\sim 1.3 \AA^{-1}$. Thus, the bands $B 1$ and $B 2$ show very complicated dis- 


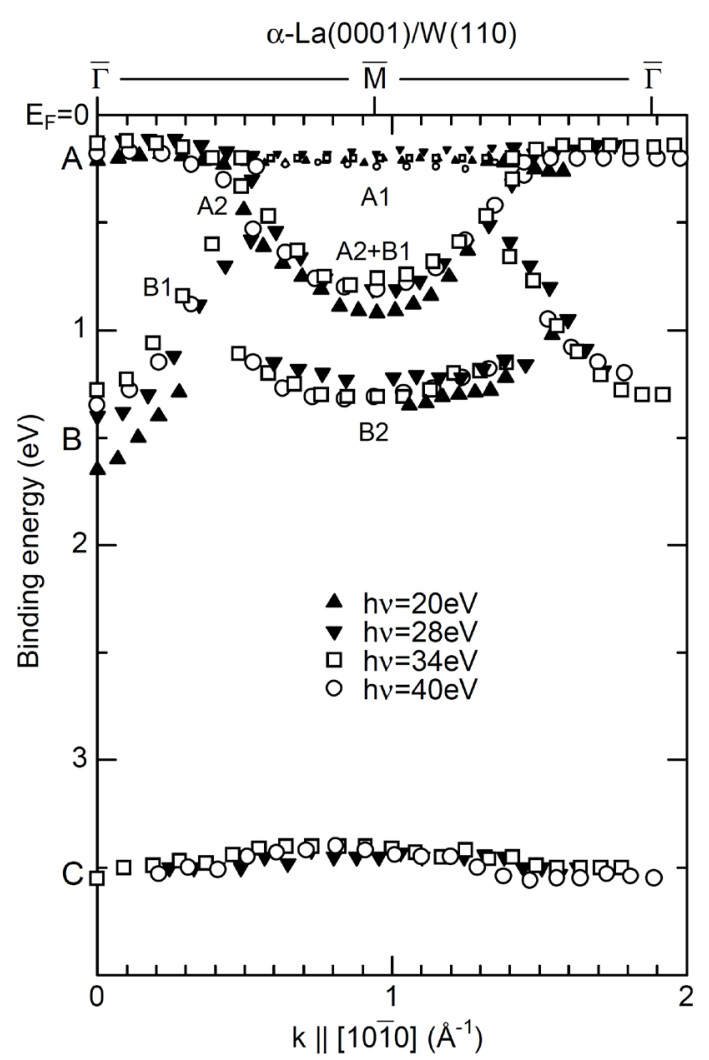

Figure 5. Measured valence-band dispersions $E\left(k_{/ /}\right)$of peaks $A(A 1, A 2), B(B 1, B 2)$, and $C$ for 4.0 - 6.0-ML $\alpha$-La(0001)/W(110) in the [10 $\overline{1} 0](\bar{\Gamma}-\bar{M})$ azimuth. Solid upward triangles, solid downward triangles, open squares, and open circles are data points for $h v=20$ $\mathrm{eV}, 28 \mathrm{eV}, 34 \mathrm{eV}$, and $40 \mathrm{eV}$ derived from Figures 4(a)-(d), respectively.

persions, up and down. Peak $C$ is weak in intensity (see Figure 4) and shows no dispersion or, if any, a very small upward dispersion by $\sim 0.1 \mathrm{eV}$ with the increase of $k_{/ /}$from 0 to $0.94 \AA^{-1}$ (peak C in Figure 4(d)).

Here, we note that the band structure is symmetrical with respect to the point of $k_{/ /}=0.94 \AA^{-1}$. If this turning $k_{/ /}$-point is $\bar{M}$ in the fcc(111) SBZ, the corresponding lattice constant is estimated to be $5.46 \AA$ since $k \overline{\bar{\Gamma}} \bar{M}=$ $2(2 / 3)^{1 / 2} \pi / a$. This $a$ value is larger than that for fcc $\beta$-La $\left(a_{\mathrm{fcc}}=5.3 \AA\right)$ by $3.0 \%$. If this turning $k_{/ /}$-point is $\bar{M}$ in the dhcp(0001) SBZ, the corresponding lattice constant is estimated to be $3.86 \AA$ since $k \bar{\Gamma} \bar{M}=2(1 / 3)^{1 / 2} \pi / a$. This $a$ value is larger than that for dhcp $\alpha$-La $\left(a_{\mathrm{dhcp}}=3.77 \AA\right.$ ) by $2.4 \%$. This comparison may indicate that the dhcp phase is slightly favorable, but the difference between 2.4 and 3.0 is too small to distinguish definitely between fcc La and dhcp La phases. Looking on fcc(111) as dhcp(0001), $a_{\text {fcc }} / a_{\text {dhcp }}=\sqrt{2}$. Note that $5.3 \AA / 3.77 \AA=1.41$. Therefore, the lattice-constant consideration alone cannot determine the crystal structure of the present La film.

It is the electronic energy dispersion relation $E(k)$ which determines the phase of La film. A great number of band-structure calculations of La have been reported so far, but most of them were for fcc $\beta$-phase, and for dhcp $\alpha$-phase one and only one has been reported by Jarlborg et al. [22]. All the calculated results for fcc $\beta$-phase are similar. We compare the experimental band structure (Figure 5) with calculated one for fcc La (Figure 5 in [22]) or dhcp La (Figure 6 in [22]), assuming that most of the observed bands are of bulk nature.

In the present case, as stated above, $k_{\perp}$ is not a good quantum number, and it would be expected that $k_{/ /}$-resolved structures in the one-dimensional density of bulk states ( $k_{/ /}$-resolved DOS) are observed by tuning $\theta_{\mathrm{e}}$. Singularities in the density of states (i.e., high occupied density of states) are prominent in the $k_{/ /}$-resolved DOS spectrum and therefore the $k_{/ /}$-resolved DOS spectrum may reflect, not rigorously but approximately, the $E\left(k_{/ /}\right)$ dispersion [30] [31]. The [1010] $(\bar{\Gamma}-\bar{M})$ azimuth of dhcp $(0001)$ corresponds to the $[11 \overline{2}](\bar{\Gamma}-\bar{M})$ azimuth of $\mathrm{fcc}(111)$. We first examine the band structure of $\mathrm{fcc} \beta$-La. A plane defined by the fcc(111) surface normal and the [112] direction is the $\Gamma K L U X(\overline{1} 10)$ mirror plane. The $\Gamma-K, \Gamma-L, \Gamma-X$, and $L-U$ axes are in the (110) plane, but, except for $L-U$, not parallel to the [112] direction. It is found that the calculated band structures along $\Gamma-K, \Gamma-L$, 


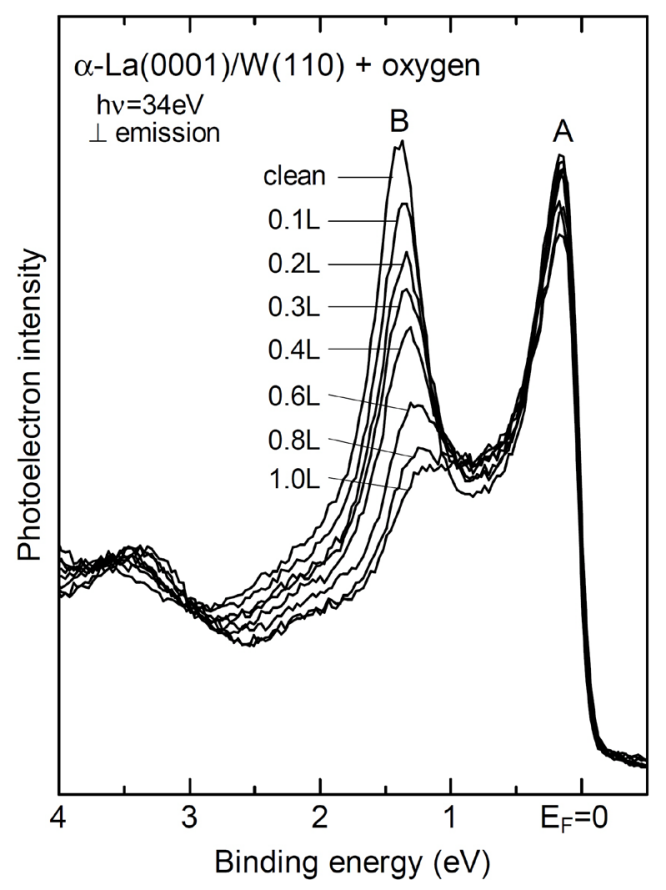

Figure 6. Comparison of the $h v=34-\mathrm{eV} 4.0-\mathrm{ML} \alpha-\mathrm{La}(0001) / \mathrm{W}(110)$ spectra before and after $\mathrm{O}_{2}$ exposure. The exposure increases from $0.1 \mathrm{~L}$ to $1.0 \mathrm{~L}$ by $0.1 \mathrm{~L}$ or $0.2 \mathrm{~L}$.

$\Gamma-X$, and $L-U$ can explain experimental band $A 2+B 1$, but are inconsistent with other bands. More important, in sharp contrast to observations, the bulk bands of fcc $\beta$-La are not symmetrical about $\bar{M}$.

In the case of dhcp $\alpha$ phase, the $(\overline{1} 2 \overline{1} 0)$ mirror plane, defined by the (0001) surface normal and the $[10 \overline{1} 0](\bar{\Gamma}$ $\bar{M})$ direction, contains the $\Gamma-M$ and $A-L$ axes, which are parallel to [10 $\overline{10]}$ and furthermore have the same one-dimensional periodicity as the $\bar{\Gamma}-\bar{M}$ axis. That is, the bulk bands along $\Gamma-M$ and $A-L$ are symmetrical about $\bar{M}$ in agreement with the observations. Comparing the experimental band structure (Figure 5) with the calculated one for dhcp La (Figure 6 in [22]), we realize fairly good correspondence between the two, with regard to position, dispersion, and periodicity. The intensity of peak $A$, which is rather weak at and around $\bar{\Gamma}$, increases with increasing $k_{/ /}$up to $\sim 0.45 \AA^{-1}$ where peak $A$ is split into two, $A 1$ and $A 2$. The reverse of this is realized from the $A 1$ - $A 2$ merging point $\left(k_{/ /} \sim 1.5 \AA^{-1}\right)$ to $\bar{\Gamma}_{2 \text { nd }}$ point in second zone $\left(k_{/ /} \sim 1.9 \AA^{-1}\right)$. Therefore, a band (or bands) should disperse down across $E_{\mathrm{F}}$ near $k_{/ /} \sim 0.45 \AA^{-1}$ and back up near $k_{/ /} \sim 1.5 \AA^{-1}$, in qualitative agreement with the calculated band structure of dhcp La [22]. Like this, a band corresponding to observed band $A 2+B 1$, $B 1$, or $B 2$ (except for $A 1$ ) is found in the calculated bands along $\Gamma-M$ or $A-L$. A band corresponding to band $A 1$ is missing: this low-intense Fermi-edge transition $A 1$ can be ascribed to a kind of DOS transition visible due to the life-time broadening of the final states [32]. Thus, as for the bands from $E_{\mathrm{F}}$ to $E_{\mathrm{B}} \sim 2 \mathrm{eV}$, which are $5 d$-like according to [22], and except for band A1, our ARPES band dispersion data are consistent with the dhcp structure rather than the fcc structure.

The band structure calculation for dhcp La predicts a 6s-like band along $\Gamma-M$ (from $E_{\mathrm{B}} \sim 2 \mathrm{eV}$ at $M$ to $\sim 3.4$ $\mathrm{eV}$ at $\Gamma$ ) [22], but is not observed in ARPES owing to its low photoionization cross section as compared with that of $5 d$. For $20 \mathrm{eV}<h v<40 \mathrm{eV}$, the Hartree-Fock-Slater atomic photoionization cross section for La $6 \mathrm{~s}$ is 10 - 30 times as small as that for La $5 d$ [25]. Note that a weak feature $C$ is observed at $E_{\mathrm{B}} \sim 3.5 \mathrm{eV}$. Band structure calculations of dhcp La predict that the bottom of $6 s$ band is at $E_{\mathrm{B}} \sim 3.4 \mathrm{eV}$ [22], in agreement with our observations. Singularities in the density of states (i.e., high occupied density of states) may lead to low-intensity structures in the spectra.

Rare earth elements are characterized by their partially filled $4 f$-shell. In the case of Ce with the ground-state electronic configuration of $[\mathrm{Xe}] 4 f^{1}(5 d 6 s)^{3}$, its valence-band photoelectron spectrum reveals a characteristic double-peaked structure: one near $E_{\mathrm{F}}$ and one at $E_{\mathrm{B}} \sim 2 \mathrm{eV}$ [23]. It is by now generally accepted that the peak near $E_{\mathrm{F}}$ is assigned to a well-screened $4 f^{1}$ final state, with the $4 f$ hole filled by a valence electron, and the other at $E_{\mathrm{B}} \sim 2 \mathrm{eV}$ is assigned to a poorly screened $4 f^{0}$ final state, with valence electrons (5d) partially screening the 
hole. Contrary to Ce, in the present case of La, the observed spectra do not show such a double-peaked structure and, e.g., a poorly-screened $4 f^{0}$ final-state feature is obviously missing. This result reconfirms that the $4 f$ hybridization with the occupied states is negligibly small in La. Furthermore, it is found that, except for $4 f$-related structures, the valence-band structure of dhcp $\alpha$-La resembles closely that of dhcp $\beta$-Ce (or fcc $\gamma$-Ce) [23].

Finally, we want to examine band $A$ and the possibility of its relation to a surface state. Recent development in valence-band photoemission studies of rare-earth metals revealed that a $d$-like surface state exists just below $E_{\mathrm{F}}$ around the center $(\bar{\Gamma})$ of the SBZ for the close-packed surfaces of almost all rare-earth metals including La [12] [14]. In fact, according to [22], there is a large gap between $E_{\mathrm{B}}=-0.5 \mathrm{eV}$ and $+1.7 \mathrm{eV}$ at and around $\vec{\Gamma}$ for dhcp $\mathrm{La}(0001)$ and, for example, according to the band calculation of an 11 layer slab for dhcp Ce, a surface state exists very close to the top of a band gap along $\bar{\Gamma}-\bar{M}$ in the SBZ (see Figure 2 in [33]). Thus, there is a possibility that band $A-A 2-A 2+B 1$ is a surface state. In order to get some insight into the character of peak $A$ and also peak $B$, we examine the influence of surface contamination on the peaks. The sensitivity to contamination is generally used as one test for surface states, as is the case of La [12]. Note that a surface state is not necessarily sensitive to the adsorption of every kind of foreign atom and some bulk state also show high sensitivity to foreign atoms [34]. Therefore, we should take notice that sensitivity to the presence of foreign atoms on the surface is not a universally correct rule for the identification of a surface state, but only a guideline. Figure 6 displays comparison of the $h v=34-\mathrm{eV} 4.0-\mathrm{ML} \alpha-\mathrm{La}(0001) / \mathrm{W}(110)$ spectra before and after $\mathrm{O}_{2}$ exposure. The exposure increases from $0.1 \mathrm{~L}$ to $1.0 \mathrm{~L}\left(1 \mathrm{~L}=1\right.$ langmuir $=10^{-6}$ Torr $\left.\cdot \mathrm{sec}\right)$. It is found that peak $B$ is rapidly quenched by exposing the $\mathrm{La}(0001)$ surface to $\mathrm{O}_{2}$ up to $1 \mathrm{~L}$, whereas peak $A$ is almost unchanged. These findings may suggest that the peak $A$ is ascribed to the bulk electronic structure of La, while the peak $B$ apparently to the surface electronic structure. However, the energy position of peak $B$ is strongly $h v$-dependent (that of peak $A$ is weakly $h v$-dependent), as clearly seen in Figure 5. Although the possibility of the existence of a surface state on dhcp $\operatorname{La}(0001)$ cannot denied completely, both peaks $A$ and $B$ are considered to be originated from the bulk-like states.

\section{Conclusion}

The electronic structure of well-ordered and atomically clean dhcp $\alpha$-La(0001) epitaxially grown on a W(110) surface has been studied by means of photoelectron spectroscopy. The photoemission cross sections of the La $5 d$ states were clarified in the $h v$ region from $20 \mathrm{eV}$ to $130 \mathrm{eV}$, confirming character of the photoemission peaks. The energy-band structure of the dhcp $\alpha$-La(0001) film was determined along the $[10 \overline{10}](\bar{\Gamma}-\bar{M})$ azimuth direction and was found to be consistent with the theoretical calculation [22]. In addition, by comparison between La and Ce, except for 4 -related structures, the valence-band structures of dhcp $\alpha$-La and dhcp $\beta$-Ce were found to resemble closely each other. Structural information of the film was obtained from the experimentally-determined dispersions: the experimental dispersions reveal a lattice constant of $3.86 \AA$, which is larger than the lattice constant of bulk dhcp $\alpha$-La only by $2.4 \%$. Contrary to expectations, no undeniable evidence for the existence of a $5 d$-like surface state near $E_{\mathrm{F}}$ at the $\bar{\Gamma}$ point of SBZ was obtained. The $6 \mathrm{~s}$ band bottom was identified. Our data demonstrated importance of using well-ordered and atomically clean $\mathrm{La}(0001)$ epitaxial films grown on a well-ordered and atomically clean W(110) surface for carrying out La photoemission experiments.

\section{Acknowledgements}

We are pleased to thank the staff of Photon Factory for their excellent support. This work has been performed under the approval of the Photon Factory Program Advisory Committee (Grant Nos. 2000G168, 2002G180, 2004G187, 2006G220, and 2008G102), and partly supported by JSPS KAKENHI Grant Number 15K04618 and Research Project from Graduate School of Science and Technology, Hirosaki University.

\section{References}

[1] Lock, J.M. (1957) The Magnetic Susceptibilities of Lanthanum, Cerium, Praseodymium, Neodymium and Samarium, from $1.5^{\circ} \mathrm{K}$ to $300^{\circ} \mathrm{K}$. Proceedings of the Physical Society, Section B, 70, 566. http://dx.doi.org/10.1088/0370-1301/70/6/304

[2] Blumberg, W.E., Eisinger, J., Jaccarino, V. and Matthias, B.T. (1960) Nuclear Magnetic Resonance in Scandium and Lanthanum Metal. Physical Review Letters, 5, 52. http://dx.doi.org/10.1103/PhysRevLett.5.52

[3] Johnson, D.L. and Finnemore, D.K. (1967) Law of Corresponding States for fcc and d-hcp La. Physical Review, 158, 
376. http://dx.doi.org/10.1103/PhysRev.158.376

[4] Andres, K. (1968) Thermal Expansion and Magnetostriction of La Metal at Low Temperatures. Physical Review, 168, 708. http://dx.doi.org/10.1103/PhysRev.168.708

[5] Lou, L.F. and Tomasch, W.J. (1972) Phonon Spectrum of La. Physical Review Letters, 29, 858. http://dx.doi.org/10.1103/PhysRevLett.29.858

[6] Wühl, H., Eichler, A. and Wittig, J. (1973) Phonon Softening in Lanthanum under Pressure. Physical Review Letters, 31, 1393. http://dx.doi.org/10.1103/PhysRevLett.31.1393

[7] Balster, H. and Wittig, J. (1975) Pressure-Induced Lattice Instability in fcc Lanthanum at Low Temperature. Journal of Low Temperature Physics, 21, 377. http://dx.doi.org/10.1007/BF01141334

[8] Wittig, J. (1984) High Pressure in Science and Technology. In: Homan, C., MacCrone, R.K. and Whalley E., Eds., High Pressure in Science and Technology, MRS Symposia Proceedings No. 22, North-Holland, Amsterdam, 17.

[9] Lang, J.K., Baer, Y. and Cox, P.A. (1981) Study of the 4f and Valence Band Density of States in Rare-Earth Metals. II. Experiment and Results. Journal of Physics F: Metal Physics, 11, 121. http://dx.doi.org/10.1088/0305-4608/11/1/015

[10] Fedorov, A.V., Laubschat, C., Starke, K., Weschke, E., Barholz, K.-U. and Kaindl, G. (1993) Surface Shift of the Unoccupied 4f State in La Metal. Physical Review Letters, 70, 1719. http://dx.doi.org/10.1103/PhysRevLett.70.1719

[11] Wieliczka, D.M., Olson, C.G. and Lynch, D.W. (1984) Valence-Band Photoemission in La and Pr: Connections with the Ce Problem. Physical Review Letters, 52, 2180-2182. http://dx.doi.org/10.1103/PhysRevLett.52.2180

[12] Fedorov, A.V., Höhr, A., Weschke, E., Starke, K., Adamchuk, A.K. and Kaindl, G. (1994) Partially Occupied Surface State at the Fermi Level of La(0001). Physical Review B, 49, 5117-5120. http://dx.doi.org/10.1103/PhysRevB.49.5117

[13] Molodtsov, S.L., Richter, M., Danzenbächer, S., Wieling, S., Steinbeck, L. and Laubschat, C. (1997) Angle-Resolved Resonant Photoemission as a Probe of Spatial Localization and Character of Electron States. Physical Review Letters, 78, 142-145. http://dx.doi.org/10.1103/PhysRevLett.78.142

[14] Weschke, E., Höhr, A., Kaindl, G., Molodtsov, S.L., Danzenbächer, S., Richter, M. and Laubschat, C. (1998) Surface Electronic Structure of Epitaxial Ce and La Films. Physical Review B, 58, 3682-3689. http://dx.doi.org/10.1103/PhysRevB.58.3682

[15] Myron, H.W. and Liu, S.H. (1970) Energy Bands for fcc Lanthanum and Praseodymium. Physical Review B, 1, 24142417. http://dx.doi.org/10.1103/PhysRevB.1.2414

[16] Duthie, J.C. and Pettifor, D.G. (1977) Correlation between d-Band Occupancy and Crystal Structure in the Rare Earths. Physical Review Letters, 38, 564-567. http://dx.doi.org/10.1103/PhysRevLett.38.564

[17] Pickett, W.E., Freeman, A.J. and Koelling, D.D. (1980) Self-Consistent Linearized Augmented-Plane-Wave Study of the Electronic Structure and Superconductivity of fcc Lanthanum under Pressure. Physical Review B, 22, 2695-2715. http://dx.doi.org/10.1103/PhysRevB.22.2695

[18] McMahan, A.K. and Young, D.A. (1984) Is There a Missing Rare Earth Crystal Structure? Physics Letters A, 105, 129131. http://dx.doi.org/10.1016/0375-9601(84)90652-2

[19] Skriver, H.L. (1985) Crystal Structure from One-Electron Theory. Physical Review B, 31, 1909-1923. http://dx.doi.org/10.1103/PhysRevB.31.1909

[20] Wang, X.W., Harmon, B.N., Chen, Y., Ho, K.-M., Stassis, C. and Weber, W. (1986) Anomalous Lattice Dynamics of fcc Lanthanum. Physical Review B, 33, 3851-3855. http://dx.doi.org/10.1103/PhysRevB.33.3851

[21] Lu, Z.W., Singh, D.J. and Krakauer, H. (1989) Ground-State Properties of fcc and bcc Lanthanum. Physical Review B, 39, 4921-4925. http://dx.doi.org/10.1103/PhysRevB.39.4921

[22] Jarlborg, T., Anderson, G., Sundqvist, B. and Rapp, O. (1989) Resistivity, Bandstructure and Superconductivity of DHCP and FCC La under Pressure. Journal of Physics: Condensed Matter, 1, 8407-8424. http://dx.doi.org/10.1088/0953-8984/1/44/012

[23] Morimoto, O., Kato, H., Enta, Y. and Sakisaka, Y. (2009) Photoemission from the Valence Bands of Ce(111) on W(110). Surface Science, 603, 2145-2151. http://dx.doi.org/10.1016/j.susc.2009.04.019

[24] Kämper, K.-P., Schmitt, W., Güntherodt, G. and Kuhlenbeck, H. (1988) Thickness Dependence of the Electronic Structure of Ultrathin, Epitaxial Ni(111)/W(110) Layers. Physical Review B, 38, 9451-9456. http://dx.doi.org/10.1103/PhysRevB.38.9451

[25] Yeh, J.J. and Lindau, I. (1985) Atomic Subshell Photoionization Cross Sections and Asymmetry Parameters: $1 \leq Z \leq$ 103. Atomic Data and Nuclear Data Tables, 32, 1-155. http://dx.doi.org/10.1016/0092-640X(85)90016-6

[26] Zimkima, T.M., Fomichev, V.A., Gribovskii, S.A. and Zhukova, I.I. (1967) Anomalies in the Character of the X-Ray Absorption of Rare-Earth Elements of the Lanthanide Group. Soviet Physics-Solid State, 9, 1128.

[27] Haensel, R., Rabe, P. and Sonntag, B. (1970) Optical Absorption of Cerium, Cerium Oxide, Praseodymium, Praseo- 
dymium Oxide, Neodymium, Neodymium Oxide and Samarium in the Extreme Ultraviolet. Solid State Communications, 8, 1845-1848. http://dx.doi.org/10.1016/0038-1098(70)90331-5

[28] Suzuki, S., Ishii, T. and Sagawa, T. (1975) 4d-Shell Photoabsorption Spectra of Lanthanum- and Cerium-Halides. Journal of the Physical Society of Japan, 38, 156-161. http://dx.doi.org/10.1143/JPSJ.38.156

[29] Aono, M., Chiang, T.C., Knapp, J.A., Tanaka, T. and Eastman, D.E. (1980) Direct Recombination and Auger Deexcitation Channels of La $4 d \rightarrow 4 f$ Resonant Excitations in $\mathrm{LaB}_{6}$. Physical Review B, 21, 2661-2665. http://dx.doi.org/10.1103/PhysRevB.21.2661

[30] Nilsson, P.O. and Dahlbäck, N. (1979) The Relation between UV-Photoemission Spectra and Bulk Band Structure: Application to Cu. Solid State Communications, 29, 303-305. http://dx.doi.org/10.1016/0038-1098(79)91062-7

[31] Gewinner, G., Peruchetti, J.C., Jaéglé, A. and Pinchaux, R. (1983) Angle-Resolved-Photoemission Study of the Cr(100) Surface. Physical Review B, 27, 3358-3365. http://dx.doi.org/10.1103/PhysRevB.27.3358

[32] Courths, R. and Hüfner, S. (1984) Photoemission Experiments on Copper. Physics Reports, 112, 53-171. http://dx.doi.org/10.1016/0370-1573(84)90167-4

[33] Schiller, F., Heber, M., Servedio, V.D.P. and Laubschat, C. (2003) Surface States and Fermi Surface of Ordered $\gamma$-Like Ce Films on W(110). Physical Review B, 68, Article ID: 233103. http://dx.doi.org/10.1103/PhysRevB.68.233103

[34] Louie, S.G., Thiry, P., Pinchaux, R., Pétroff, Y., Chandesris, D. and Lecante, J. (1980) Periodic Oscillations of the Frequency-Dependent Photoelectric Cross Sections of Surface States: Theory and Experiment. Physical Review Letters, 44, 549-553. http://dx.doi.org/10.1103/PhysRevLett.44.549 